\title{
Phytotherapie bei Ferkelkrankheiten - Möglichkeiten und Grenzen der Behandlung
}

\author{
Werner Hagmüller \\ Höhere Bundeslehr- und Forschungsanstalt für Landwirtschaft Raumberg-Gumpenstein, Aussenstelle Wels, Thalheim bei Wels, Österreich
}

\section{Einleitung}

Der Einsatz von antimikrobiell wirksamen Substanzen beim Schwein erfolgt hauptsächlich in Form von metaphylaktischen oder therapeutischen Massnahmen [1]. Da bei grossen Tierzahlen das Individuum immer stärker in den Hintergrund tritt, steht in der Behandlung von Schweinen die Herdendiagnostik und -therapie im Vordergrund.

Das Bundesamt für Verbraucherschutz und Lebensmittelsicherheit in Deutschland hat im Frühjahr 2015 erste Zahlen des Antibiotikamonitorings bekannt gegeben. Aus diesem Bericht wird ersichtlich, dass neben dem Geflügel Ferkel bis $30 \mathrm{~kg}$ Körpergewicht die am häufigsten therapierte Gruppe darstellen (Tab. 1) [2].

Indikationen für die Behandlung von Ferkeln mit Antibiotika sind vor allem Infektionen mit Streptokokken, Staphylokokken, Escherichia coli, Pasteurella spp., Mycoplasma spp., Actinobacillus pleuropneumoniae, Brachyspira hyodysenteriae sowie Lawsonia intracellularis [1]. Dabei spielen parenterale Verabreichungen von lang wirksamen Präparaten vor allem in den ersten Lebenstagen der Saugferkel als Schutz gegen Streptokokken oder Mycoplasma hyorhinis eine bedeutende Rolle [3, 4].

\section{Phytotherapeutische Behandlung}

Die Therapie mit Pflanzen, Pflanzenteilen oder deren Zubereitungen (z.B. Extrakte) versteht sich als ein Teil der Schulmedizin, wird also zu den allopathischen Behandlungsmethoden gezählt. Während in der Gesunderhaltung und Behandlung von Rindern phytotherapeutisches Wissen in der bäuerlichen Bevölkerung noch vorhanden ist $[5,6]$, gibt es beim Schwein nur wenig überliefertes Wissen.

In der Literatur der letzten 20 Jahre finden sich viele Berichte über den Einsatz von Pflanzen und pflanzlichen Substanzen. Diese zielen jedoch nicht auf die Behandlung von Krankheiten, sondern auf die Verbesserung der Verdauungsfunktion $\mathrm{ab}$ und sind im weitesten Sinne dem Thema «natürliche Leistungsförderung» zuzuordnen [7-9].
Tab. 1. Kennzahlen zur Therapiehäufigkeit (Therapiehäufigkeitsindex = Anzahl behandelter Tiere multipliziert mit der Anzahl Behandlungstage dividiert durch die durchschnittliche Anzahl gehaltener Tiere pro Halbjahr)

\begin{tabular}{lcc}
\hline Tierart/Nutzungsart & Median & Drittes Quartil \\
\hline Mastkälber bis 8 Monate & 0,000 & 5,058 \\
Mastrinder älter als 8 Monate & 0,000 & 0,015 \\
Ferkel bis 30 kg Körpergewicht & 4,793 & 26,191 \\
Mastschweine über 30 kg Körpergewicht & 1,199 & 9,491 \\
Masthühner & 19,558 & 35,032 \\
Mastputen & 23,030 & 47,486 \\
\hline
\end{tabular}

Ferkel werden je nach Wirtschaftsweise zwischen 4 und 6 Wochen gesäugt. Während der Säugezeit sind phytotherapeutische Behandlungen schwieriger durchzuführen als nach dem Absetzen, da Saugferkel neben der Muttermilch nur geringe Mengen an Wasser und Beifutter aufnehmen. Dadurch sind Medikationen über Wasser und Futter kaum möglich. Akute Saugferkelerkrankungen (Coli-Diarrhö, Clostridienenteritis, Streptokokkenmeningitis, Gelenkentzündungen) werden deshalb fast ausschliesslich mit chemisch-synthetischen Arzneimitteln behandelt. Die Hauptindikationen für den Einsatz von Phytotherapeutika bei Ferkeln beschränken sich also auf die Zeit nach dem Absetzen und betreffen vorwiegend Durchfälle und Atemwegserkrankungen.

\section{Durchfallerkrankungen}

Die Phase unmittelbar nach dem Absetzen der Ferkel vom Muttertier führt häufig zu Enteritiden, die vor allem durch unterschiedliche Stämme von E. coli verursacht werden. Diese heften sich an der Darmschleimhaut an und kehren die osmotischen Verhältnisse in den Darmepithelzellen um. Damit werden vermehrt Wasser und Elektrolyte in das Darmlumen sezerniert [10]. Die Ferkel zeigen vorerst noch normale Fresslust - bei hochgradigem, wässrigem Durchfall. Ohne Behandlung führt die Krankheit bis zum Tod des Tieres durch Dehydratation.

\section{KARGER}

Fax +497614520714

Information@Karger.com

www.karger.com
Dr. Werner Hagmüller

Höhere Bundeslehr- und Forschungsanstalt für Landwirtschaft RaumbergGumpenstein, Aussenstelle Wels

Austrasse 10, 4600 Thalheim bei Wels, Österreich

werner.hagmueller@raumberg-gumpenstein.at 


\section{Pektine}

Oligosaccharide, die aus Pektin gewonnen werden, können die Anheftung von E. coli an der Darmwand verhindern. Somit werden die Bakterien über den Verdauungsbrei weitergeleitet und ausgeschieden $[11,12]$. Weiterhin führen Pektine zu einer absorptiven Bindung von Toxinen und zur Absenkung des pH-Werts im Darm [13]. Als bekannteste Pflanze ist die Karotte (Daucus carota L.) zu nennen, die bereits zu Beginn des 20. Jahrhunderts in der Kinderheilkunde als bewährtes Durchfallmittel in Form der Moro'schen Karottensuppe eingesetzt wurde [14].

\section{Gerbstoffe}

Sie besitzen neben einer direkt antiinfektiösen Wirkung auch eine schleimhautabdichtende und sekretionshemmende Wirkung [13]. Da Gerbstoffe für Ferkel nicht attraktiv sind, ist die Verabreichung als Tee oder Dekokt [15] im Gemisch mit Elektrolytlösungen zu empfehlen. Zu den häufig verwendeten Gerbstoffdrogen zählen Blutwurz (Potentilla erecta), Eichenrinde (Quercus robur) und Heidelbeere (Vaccinium myrtillus). Eichenrinde konnte in einem Versuch mit Absetzferkeln in Kombination mit rationierter Fütterung die Kotkonsistenz verbessern [16].

\section{Atemwegserkrankungen}

Die Behandlung von bakteriell oder viral bedingten Atemwegserkrankungen erfolgt vornehmlich über die Verabreichung von Teedrogen. Tees werden von Ferkeln - sofern sie gesüsst werden - gerne und auch in ausreichender Menge aufgenommen. Eine einfache Teemischung stellt der Brusttee (Species pectorales ÖAB) dar. Species pectorales enthält Malvenblüte, Eibischblatt, Thymiankraut, Süssholzwurzel, Königskerze, Eibischwurzel sowie Anisfrüchte und dient zur Förderung der Schleimsekretion und Reizlinderung bei Erkrankungen der Atemwege. Bei einem Influenzaausbruch in einem Schweinemaststall konnte durch die alleinige Behandlung der Tiere mit Brusttee völlig auf chemisch-synthetische Arzneimittel verzichtet werden [17].

\section{Rechtliche Aspekte}

Die Anwendung von Heilpflanzen in der Nutztierhaltung kann auf drei Arten erfolgen:

(1) Phytotherapeutika: Hierunter werden zugelassene pflanzliche Arzneimittel verstanden. Sie unterliegen dem Arzneimittelgesetz (AMG) und werden entweder als Human- oder als Veterinärarzneispezialitäten bezeichnet. Die Anzahl verfügbarer Phytotherapeutika für lebensmittelliefernde Nutztiere in Österreich ist gering (ca. 10 Präparate).

(2) Futtermittel: Diese unterliegen dem Futtermittelgesetz; ihre Verabreichung dient aber in erster Linie der Ernährung. Zur Pflege und Gesunderhaltung werden Heilpflanzen trotzdem von Tierärzten und Landwirten eingesetzt. Am Beispiel von Kamillentee soll die komplexe Rechtsmaterie erklärt werden: Ein Landwirt darf Kamille als Einzelfuttermittel mit heissem Wasser mischen und dem Tier verabreichen. Dies stellt jedoch keine Behandlung im Sinne des AMG dar. Demnach kann auch der Tierarzt Kamillentee nicht als Medikament verabreichen.

(3) Hausmittel: Diese werden zur Pflege des gesunden Tieres oder zur Verbesserung der Befindlichkeit verwendet [18]. Die Zubereitung erfolgt nach traditionellen Überlieferungen sowie regional unterschiedlich; deshalb ist eine objektive Bewertung schwierig.

\section{Fazit}

Eine phytotherapeutische Behandlung von Ferkeln erfolgt in erster Linie in der Zeit zwischen Absetzen und Verkauf an den Mastbetrieb (Gewichtsbereich 10-30 kg). Einzeltierbehandlungen sind selten; hauptsächlich werden ganze Würfe bzw. ganze Stallabteile behandelt. Da nur wenige Fertigarzneimittel zur Verfügung stehen - im Ferkelbereich nur etwa 5 Präparate -, erfolgt eine «Behandlung» häufig in Form von Futtermitteln oder Hausmitteln durch die Tierhalter. Zur Förderung der Phytotherapie und gleichzeitigen Verringerung des Antibiotikaverbrauchs bei lebensmittelliefernden Nutztieren ist eine Änderung der Rechtslage nötig. Solange es für Tierärztinnen und Tierärzte einfacher ist, Antibiotika zu verschreiben, wird Phytotherapie in der Nutztierhaltung weiterhin ein Nischendasein fristen.

\section{Online Supplemental Material}

Die Literatur findet sich im Tagungsband und kann auf www.smgp.ch/ smgp/homeindex/jahrestagung.html heruntergeladen werden.

\section{Disclosure Statement}

Kein Interessenkonflikt. 